\title{
ON MAHLER'S CLASSIFICATION OF FORMAL POWER SERIES OVER A FINITE FIELD
}

\author{
GÜLCAN KEKEÇ \\ (Communicated by István Gaál)
}

\begin{abstract}
Let $K$ be a finite field, $K(x)$ be the field of rational functions in $x$ over $K$ and $\mathbb{K}$ be the field of formal power series over $K$. We show that under certain conditions integral combinations with algebraic formal power series coefficients of a $U_{1}$-number in $\mathbb{K}$ are $U_{m}$-numbers in $\mathbb{K}$, where $m$ is the degree of the algebraic extension of $K(x)$, determined by these algebraic formal power series coefficients.
\end{abstract}

$$
\begin{gathered}
\text { (C) } 2022 \\
\text { Mathematical Institute } \\
\text { Slovak Academy of Sciences }
\end{gathered}
$$

\section{Introduction}

\subsection{The field of formal power series over a finite field}

Let $K$ be a finite field with $q$ elements. We denote the ring of polynomials in $x$ with coefficients from $K$ by $K[x]$, the quotient field of $K[x]$ by $K(x)$ and the degree of a non-zero polynomial $f(x)$ in $K[x]$ by $\operatorname{deg}(f)$. A non-Archimedean absolute value $|\cdot|$ is defined on $K(x)$ by setting

$$
|0|=0 \quad \text { and } \quad\left|\frac{f(x)}{g(x)}\right|=q^{\operatorname{deg}(f)-\operatorname{deg}(g)},
$$

where $f(x)$ and $g(x)$ are non-zero polynomials in $K[x]$. The field $\mathbb{K}$ of formal power series over $K$ is the completion of $K(x)$ with respect to $|\cdot|$. We denote the unique extension of $|\cdot|$ to the field $\mathbb{K}$ by the same notation $|\cdot|$. Every non-zero element $\xi$ of $\mathbb{K}$ can be represented uniquely as

$$
\xi=\sum_{n=r}^{\infty} a_{n} x^{-n},
$$

where $a_{n} \in K(n=r, r+1, \ldots)$ with $a_{r} \neq 0$ and $r$ is the rational integer such that $|\xi|=q^{-r}$.

An element of $\mathbb{K}$ is called an algebraic (resp. a transcendental) formal power series if it is algebraic (resp. transcendental) over $K(x)$. We regard $K[x], K(x)$ and $\mathbb{K}$ as the analogues of $\mathbb{Z}$, $\mathbb{Q}$ and $\mathbb{R}$, respectively.

Let $P(y)=f_{0}+f_{1} y+\cdots+f_{n} y^{n}$ be a non-zero polynomial in $y$ over $K[x]$. (Note that $f_{i} \in K[x]$ for $i=0,1, \ldots, n$.) We denote the degree of $P(y)$ with respect to $y$ by $\operatorname{deg}(P)$ and the height of $P(y)$ by $H(P)$, where $H(P)=\max \left\{\left|f_{0}\right|, \ldots,\left|f_{n}\right|\right\}$. Let $\alpha$ be an algebraic formal power series and $P(y)$ be its minimal polynomial over $K[x]$. Then the height $H(\alpha)$ and the degree $\operatorname{deg}(\alpha)$ of $\alpha$ are defined by $H(P)$ and $\operatorname{deg}(P)$, respectively. Moreover, the roots of $P(y)$ are said to be the conjugates of $\alpha$ over $K(x)$. We refer the reader to Sprindžuk 16 for detailed information on $\mathbb{K}$.

2010 Mathematics Subject Classification: Primary 11J61; Secondary 11J82.

Keywords: Mahler's classification of formal power series over a finite field, $U$-number, transcendence measure. 


\section{GÜLCAN KEKEC}

\subsection{Mahler's classification of formal power series over a finite field}

In 1932, Mahler 9 introduced a classification of complex numbers. He divided complex numbers into four disjoint classes and called the numbers in these classes $A-, S$-, $T$ - and $U$-numbers. Two algebraically dependent complex numbers belong to the same class. The class of $A$-numbers coincides with the set of complex algebraic numbers. Almost all complex numbers, in the sense of Lebesgue measure on $\mathbb{C}$, are $S$-numbers. Schmidt 15 proved that there exist uncountably many $T$-numbers. The class of $U$-numbers is subdivided into $U_{m}$-subclasses $(m=1,2,3, \ldots)$. LeVeque [8] constructed the first explicit examples of $U_{m}$-numbers for any positive integer $m$.

Let $p$ be a prime number. The field $\mathbb{Q}_{p}$ of $p$-adic numbers is the completion of $\mathbb{Q}$ with respect to the $p$-adic absolute value. In 1935, Mahler 10 proposed a classification of $p$-adic numbers, analogous with his classification of complex numbers. He divided $p$-adic numbers into four disjoint classes and called the numbers in these classes $p$-adic $A$-, $S$-, $T$ - and $U$-numbers. Two algebraically dependent $p$-adic numbers belong to the same class. The class of $p$-adic $A$-numbers coincides with the set of $p$-adic algebraic numbers. Almost all $p$-adic numbers, in the sense of Haar measure on $\mathbb{Q}_{p}$, are $p$-adic $S$-numbers. Schlickewei $[14$ proved that there exist $p$-adic $T$-numbers. The class of $p$-adic $U$-numbers is subdivided into $U_{m}$-subclasses $(m=1,2,3, \ldots)$. Alnıaçık 1: Chapter III, Theorem I] constructed the first explicit examples of $p$-adic $U_{m}$-numbers for any positive integer $m$. We refer the reader to Bugeaud [2] for further information and references on Mahler's classification of complex numbers and of $p$-adic numbers.

In 1978, Bundschuh 3 introduced a classification in $\mathbb{K}$ in analogy with Mahler's classification of complex numbers and of $p$-adic numbers. This classification is said to be Mahler's classification of formal power series over a finite field. Formal power series are divided into four disjoint classes with respect to this classification, called $A-, S$-, $T$ - and $U$-numbers as follows.

Let $\xi \in \mathbb{K}$ be any formal power series over $K$ and let $n$ and $H$ be any positive rational integers. Set

$$
\begin{gathered}
w_{n}(H, \xi)=\min \{|P(\xi)|: P(y) \in K[x][y], \operatorname{deg}(P) \leq n, H(P) \leq H \text { and } P(\xi) \neq 0\}, \\
w_{n}(\xi)=\limsup _{H \rightarrow \infty} \frac{-\log w_{n}(H, \xi)}{\log H} \quad \text { and } \quad w(\xi)=\limsup _{n \rightarrow \infty} \frac{w_{n}(\xi)}{n} .
\end{gathered}
$$

We denote by $\mu(\xi)$ the smallest integer $n$ such that $w_{n}(\xi)=\infty$ if such an integer exists and write $\mu(\xi)=\infty$ otherwise. Then $\xi$ is said to be

- an $A$-number if $w(\xi)=0$ and $\mu(\xi)=\infty$,

- an $S$-number if $0<w(\xi)<\infty$ and $\mu(\xi)=\infty$,

- a $T$-number if $w(\xi)=\infty$ and $\mu(\xi)=\infty$,

- a $U$-number if $w(\xi)=\infty$ and $\mu(\xi)<\infty$.

Furthermore, a $U$-number $\xi$ is said to be a $U_{m}$-number if $\mu(\xi)=m$.

Recently, Ooto [11] contributed a lot to Mahler's classification in $\mathbb{K}$, stated as follows. The class of $A$-numbers coincides with the set of algebraic formal power series (Ooto [11: Proposition A.3]). Two algebraically dependent transcendental formal power series belong to the same class (Ooto 11. Proposition A.4]). Sprindžuk 16: Part II, Chapter 3] proved that almost all formal power series $\xi$ in $\mathbb{K}$, in the sense of Haar measure on $\mathbb{K}$, are $S$-numbers with $w_{n}(\xi)=n$ for $n=1,2,3, \ldots$ and hence with $w(\xi)=1$. Ooto [11. Theorem 1.1] recently proved that there exist uncountably many $T$-numbers in $\mathbb{K}$. He constructed explicit examples of $T$-numbers in $\mathbb{K}$. The existence of $U_{m}$-numbers for any positive integer $m$ was first proved by Oryan [12]. His method allows us to construct explicit examples of $U_{m}$-numbers in $\mathbb{K}$. Recently, Kekeç [6] and [7] contributed to constructing explicit examples of $U_{m}$-numbers in $\mathbb{K}$ by making use of the method of Oryan 12 
Satz 4 and Satz 5]. We recommend the reader to consult Sprindžuk [16], Bundschuh [3] and Bugeaud $[2]$ for further results and references on Mahler's classification in $\mathbb{K}$.

\subsection{Construction of our main result}

In 1979, Alnıaçı [1. Chapter III, pp. 73-81] proved that under certain conditions rational and integral combinations with $p$-adic algebraic number coefficients of a $p$-adic $U_{1}$-number are $p$-adic $U_{m}$-numbers, where $m$ is the degree of the $p$-adic algebraic number field, determined by these $p$-adic algebraic number coefficients. By this method, Alnıaçık proved the existence of $p$-adic $U_{m^{-}}$ numbers and gave the first explicit construction of such numbers. In the sense of this result and method of Alnıaçık, we prove the following theorem. Our method heavily depends on the method given by Oryan [12. Satz 4 and Satz 5, pp. 57-62] to prove the existence of $U_{m}$-numbers in $\mathbb{K}$. We state our main result as follows.

THEOREM 1.1. Let $\alpha_{0}, \ldots, \alpha_{k}(k \geq 1)$ be algebraic formal power series with $\alpha_{k} \neq 0$, and let $\xi$ be a $U_{1}$-number enjoying such a representation

$$
\xi=\sum_{n=0}^{\infty} a_{n} x^{-u_{n}}
$$

where $a_{n} \in K-\{0\}(n=0,1,2, \ldots)$ and $\left\{u_{n}\right\}_{n=0}^{\infty}$ is a strictly increasing sequence of non-negative rational integers with

$$
\lim _{n \rightarrow \infty} \frac{u_{n+1}}{u_{n}}=\infty
$$

Then $\alpha_{0}+\alpha_{1} \xi+\cdots+\alpha_{k} \xi^{k}$ is a $U_{m}$-number, where $m$ is the degree of $K(x)\left(\alpha_{0}, \ldots, \alpha_{k}\right)$ over $K(x)$.

Remark 1. By definition, as described in Subsection 1.2 a $U_{1}$-number $\xi$ in $\mathbb{K}$ is a formal power series $\xi$ in $\mathbb{K}$, enjoying such an approximation

$$
0<\left|\xi-\frac{a_{n}(x)}{b_{n}(x)}\right|<H\left(\frac{a_{n}(x)}{b_{n}(x)}\right)^{-d_{n}} \quad(n=1,2,3, \ldots)
$$

where $a_{n}(x), b_{n}(x)$ are non-zero polynomials in $K[x]$ with $H\left(a_{n}(x) / b_{n}(x)\right)>1$ for $n=1,2,3, \ldots$ and $\left\{d_{n}\right\}_{n=1}^{\infty}$ is a sequence of positive real numbers with $\lim _{n \rightarrow \infty} d_{n}=\infty$. Therefore the representation (1.1) together with $(1.2)$ is not a characterization of a $U_{1}$-number. The set of numbers $\xi$ satisfying (1.1) and 1.2 is just a subset of the set of $U_{1}$-numbers. Moreover, we see from the definition that a $U_{1}$-number in $\mathbb{K}$ is the analogue of a Liouville number in $\mathbb{R}$. We refer the reader to Perron 13 for information on Liouville numbers.

Remark 2. Theorem 1.1 is the $\mathbb{K}$ analogue of [1: Chapter III, Theorem I] in $p$-adic case and of [5: Corollary 1] in real case. Furthermore, we recommend the reader to consult a related work [4] in real case, which yields to construct explicit $U_{m}$-numbers in $\mathbb{R}$.

We highlight the following consequence of Theorem 1.1 .

Corollary 1.1.1. Let $\alpha \in \mathbb{K}-\{0\}$ be algebraic over $K(x)$ with $\operatorname{deg}(\alpha)=m$ and $\xi$ be a $U_{1}$-number satisfying the conditions of Theorem 1.1. Then, by Theorem 1.1, $\alpha+\xi$ and $\alpha \cdot \xi$ are $U_{m}$-numbers.

In the next section, we prepare and cite certain lemmas in order to prove Theorem 1.1. In Section 3, we prove Theorem 1.1 and establish explicit examples of $U_{m}$-numbers. 


\section{GÜLCAN KEKEC}

\section{Auxiliary results}

We apply the following four lemmas to prove Theorem 1.1. Lemma 2.1 can be regarded as a $\mathbb{K}$ analogue of Lemma 5 in [1: Chapter I].

LEмма 2.1. Let $\alpha_{0}, \ldots, \alpha_{k}(k \geq 1)$ be algebraic formal power series with $\alpha_{k} \neq 0$. Then for $\theta$ in $K(x)$ the algebraic formal power series $\alpha_{0}+\alpha_{1} \theta+\cdots+\alpha_{k} \theta^{k}$ is a primitive element of $K(x)\left(\alpha_{0}, \ldots, \alpha_{k}\right)$ over $K(x)$ except for only finitely many $\theta$ in $K(x)$.

Pr o of. We adapt the method of the proof of Lemma 5 in 1 : Chapter I] to the field $\overline{K(x)}$, where $\overline{K(x)}$ denotes the algebraic closure of $K(x)$. The field $\overline{K(x)}$ is the analogue of the field $\overline{\mathbb{Q}}$ of complex algebraic numbers. We put

$$
L:=K(x)\left(\alpha_{0}, \ldots, \alpha_{k}\right)
$$

and

$$
R(y):=\alpha_{0}+\alpha_{1} y+\cdots+\alpha_{k} y^{k} .
$$

Let us denote the degree of $L$ over $K(x)$ by $m$. If $m=1$, then Lemma 2.1 obviously holds true. Hence, let us assume that $m>1$. We denote by $\alpha_{i}^{(1)}, \ldots, \alpha_{i}^{(m)}$ the field conjugates of $\alpha_{i}$ for $L$, where $i=0,1, \ldots, k$. These are the conjugates of $\alpha_{i}$ over $K(x)$, each repeated $m / \operatorname{deg}\left(\alpha_{i}\right)$ times. We exclude the values $\theta \in K(x)$ satisfying $R(\theta)=0$, if such values of $\theta$ exist, since they constitute a finite set. Let $\theta \in K(x)$ with $R(\theta) \neq 0$. If $R(\theta)$ is not a primitive element of $L$, then there exist integers $s, t$ in $\{1, \ldots, m\}$ with $s \neq t$ such that

$$
(R(\theta))^{(s)}=(R(\theta))^{(t)}
$$

From 2.1],

$$
\left(\alpha_{0}+\alpha_{1} \theta+\cdots+\alpha_{k} \theta^{k}\right)^{(s)}=\left(\alpha_{0}+\alpha_{1} \theta+\cdots+\alpha_{k} \theta^{k}\right)^{(t)} .
$$

This gives

$$
\alpha_{0}^{(s)}+\alpha_{1}^{(s)} \theta^{(s)}+\cdots+\alpha_{k}^{(s)}\left(\theta^{k}\right)^{(s)}=\alpha_{0}^{(t)}+\alpha_{1}^{(t)} \theta^{(t)}+\cdots+\alpha_{k}^{(t)}\left(\theta^{k}\right)^{(t)}
$$

and hence

$$
\alpha_{0}^{(s)}+\alpha_{1}^{(s)} \theta^{(s)}+\cdots+\alpha_{k}^{(s)}\left(\theta^{(s)}\right)^{k}=\alpha_{0}^{(t)}+\alpha_{1}^{(t)} \theta^{(t)}+\cdots+\alpha_{k}^{(t)}\left(\theta^{(t)}\right)^{k} .
$$

Since $\theta \in K(x)$, we have $\theta^{(s)}=\theta^{(t)}=\theta$. Thus

$$
\alpha_{0}^{(s)}+\alpha_{1}^{(s)} \theta+\cdots+\alpha_{k}^{(s)} \theta^{k}=\alpha_{0}^{(t)}+\alpha_{1}^{(t)} \theta+\cdots+\alpha_{k}^{(t)} \theta^{k} .
$$

If (2.1) were satisfied by infinitely many $\theta$ in $K(x)$, then the last equality would turn to an identity and we would have

$$
\alpha_{i}^{(s)}=\alpha_{i}^{(t)} \quad(i=0,1, \ldots, k) .
$$

Let $\beta$ be a primitive element of $L$ over $K(x)$. Then $\operatorname{deg}(\beta)=m$ and the field conjugates of $\beta$ for $L$ are distinct from each other. We have

$$
\beta=S\left(\alpha_{0}, \alpha_{1}, \ldots, \alpha_{k}\right),
$$

where $S\left(y_{0}, y_{1}, \ldots, y_{k}\right)$ is a rational function in $y_{0}, y_{1}, \ldots, y_{k}$ with coefficients from $K(x)$. Then we obtain

for $\nu=1,2, \ldots, m$. By 2.2, this would give us

$$
\beta^{(\nu)}=S\left(\alpha_{0}^{(\nu)}, \alpha_{1}^{(\nu)}, \ldots, \alpha_{k}^{(\nu)}\right)
$$

$$
\beta^{(s)}=\beta^{(t)} .
$$

But this is impossible since $\beta$ is a primitive element of $L$. Therefore 2.1 can hold true for only finitely many $\theta$ in $K(x)$. This completes the proof of Lemma 2.1. 
Lemma 2.2 (Oryan 12 Hilfssatz 4]). Let $\beta_{1}, \ldots, \beta_{n}$ be in a finite extension of degree $m$ over $K(x)$. Then

and

$$
H\left(\beta_{1}+\cdots+\beta_{n}\right) \leq H\left(\beta_{1}\right)^{2 m^{2}} \cdots H\left(\beta_{n}\right)^{2 m^{2}}
$$

$$
H\left(\beta_{1} \cdots \beta_{n}\right) \leq H\left(\beta_{1}\right)^{2 m^{2}} \cdots H\left(\beta_{n}\right)^{2 m^{2}} .
$$

In classical case, it is well-known that the inequality $|\beta| \leq H(\beta)+1$ holds for a complex algebraic number $\beta$, where $H(\beta)$ denotes its usual height and $|\cdot|$ denotes the usual absolute value on $\mathbb{C}$. The following lemma is the $\mathbb{K}$ analogue of this well-known result, whose proof can be done easily by following the lines in Waldschmidt [17: 3.5 Liouville's Inequalities, p. 82] and using the property of non-Archimedean absolute value.

Leмma 2.3. Let $\beta$ be an algebraic formal power series. Then

$$
|\beta| \leq H(\beta) \text {. }
$$

Lemma 2.4 (Oryan [12], Hilfssatz 2). Let $P(y)$ and $Q(y)$ be polynomials over $K[x]$ with degrees $n \geq 1$ and $m \geq 2$, respectively. If the polynomials $P(y)$ and $Q(y)$ are relatively prime over $K[x]$ and $\alpha$ is a root of $Q(y)$, then

$$
|P(\alpha)| \geq H(P)^{-m+1} H(Q)^{-n}
$$

\section{Proof of Theorem 1.1}

We write

$$
\xi=\xi_{n}+\rho_{n} \quad(n=0,1,2, \ldots)
$$

where

$$
\xi_{n}=\sum_{h=0}^{n} a_{h} x^{-u_{h}} \quad \text { and } \quad \rho_{n}=\sum_{h=n+1}^{\infty} a_{h} x^{-u_{h}} \quad(n=0,1,2, \ldots) .
$$

It follows that

$$
|\xi|=\left|\xi_{n}\right|=q^{-u_{0}} \leq 1 \quad(n=0,1,2, \ldots)
$$

and

We put

$$
\left|\rho_{n}\right|=q^{-u_{n+1}}<1 \quad(n=0,1,2, \ldots) .
$$

We want to show that $\gamma$ is a $U_{m}$-number. Set

$$
\gamma=R\left(\xi_{n}+\rho_{n}\right)=\gamma_{n}+\rho_{n} \delta_{n}
$$

where

and

$$
\gamma_{n}=R\left(\xi_{n}\right) \quad(n=0,1,2, \ldots)
$$

$$
\delta_{n}=\alpha_{1}+\alpha_{2}\left(2 \xi_{n}+\rho_{n}\right)+\cdots+\alpha_{k}\left(\left(\begin{array}{l}
k \\
1
\end{array}\right) \xi_{n}^{k-1}+\left(\begin{array}{l}
k \\
2
\end{array}\right) \xi_{n}^{k-2} \rho_{n}+\cdots+\rho_{n}^{k-1}\right)
$$

for $n=0,1,2, \ldots$. Let

$$
\left|\alpha_{i}\right|=: q^{-t_{i}} \quad(i=1, \ldots, k) \quad \text { and } \quad t:=\min \left\{0, t_{1}, \ldots, t_{k}\right\} .
$$

Then, using (3.1), 3.2 and the property of non-Archimedean absolute value, that is, $|\alpha+\beta| \leq$ $\max \{|\alpha|,|\beta|\}$ holds for $\alpha, \beta \in \mathbb{K}$, we get

$$
\left|\delta_{n}\right| \leq q^{-t} \quad(n=0,1,2, \ldots)
$$




\section{GÜLCAN KEKEC}

Note that $\gamma_{n} \in K(x)\left(\alpha_{0}, \ldots, \alpha_{k}\right)$ for $n=0,1,2, \ldots$ We deduce from Lemma 2.1 that $\operatorname{deg}\left(\gamma_{n}\right)=m$ and further from Lemma 2.2 the fact $H\left(\xi_{n}\right)=q^{u_{n}}(n=0,1,2, \ldots)$ that

$$
H\left(\gamma_{n}\right) \leq c_{0}^{u_{n}}
$$

hold for sufficiently large $n$, where $c_{0}$ is a real constant which depends only on $m, q, k$ and the algebraic formal power series $\alpha_{i}(i=0,1, \ldots, k)$ with $c_{0}>q$.

We shall first show that $\gamma$ is a $U$-number with $\mu(\gamma) \leq m$. Let $A_{n}(y)=a_{n 0}+a_{n 1} y+\cdots+a_{n m} y^{m}$ be the minimal polynomial of $\gamma_{n}$ over $K[x]$. Since $A_{n}\left(\gamma_{n}\right)=0$, we have

$$
A_{n}(\gamma)=A_{n}\left(\gamma_{n}+\rho_{n} \delta_{n}\right)=\rho_{n} \sigma_{n}
$$

where

$$
\sigma_{n}=a_{n 1} \delta_{n}+\cdots+a_{n m}\left(\left(\begin{array}{c}
m \\
1
\end{array}\right) \gamma_{n}^{m-1} \delta_{n}+\left(\begin{array}{c}
m \\
2
\end{array}\right) \gamma_{n}^{m-2} \rho_{n} \delta_{n}^{2}+\cdots+\rho_{n}^{m-1} \delta_{n}^{m}\right) .
$$

It follows from Lemma 2.3, (3.2), (3.3) and 3.4) that

$$
\left|\sigma_{n}\right| \leq c_{1}^{u_{n}}
$$

where $c_{1}$ is a real constant with $c_{0}<c_{1}$, thus from 3.2 that

$$
\left|A_{n}(\gamma)\right|=\left|\rho_{n}\right|\left|\sigma_{n}\right| \leq q^{-u_{n+1}} c_{1}^{u_{n}}
$$

and hence from (3.4) that

$$
0<\left|A_{n}(\gamma)\right| \leq H\left(\gamma_{n}\right)^{-s_{n}}
$$

hold for sufficiently large $n$, where

$$
s_{n}=\frac{u_{n+1}}{u_{n}} \frac{\log q}{\log c_{1}}-1 \quad \text { and } \quad \lim _{n \rightarrow \infty} s_{n}=\infty .
$$

Since $\xi$ is a transcendental formal power series, note that $A_{n}(\gamma)=A_{n}(R(\xi))$ is not zero. The sequence $\left\{A_{n}(y)\right\}_{n=0}^{\infty}$ has infinitely many different terms. For otherwise $\left\{A_{n}(y)\right\}_{n=0}^{\infty}$ would have finitely many different terms, that is, there would exist a repeating polynomial $A(y)$ among these terms. Namely, there would exist a subsequence $\left\{A_{n_{j}}(y)\right\}_{j=0}^{\infty}$ of $\left\{A_{n}(y)\right\}_{n=0}^{\infty}$ such that $A_{n_{j}}(y)=$ $A(y)$ for $j=0,1,2, \ldots$. Since $A_{n_{j}}(y)$ is the minimal polynomial of $\gamma_{n_{j}}=R\left(\xi_{n_{j}}\right)$ for $j=0,1,2, \ldots$, we would obtain

$$
A\left(R\left(\xi_{n_{j}}\right)\right)=0 \quad(j=0,1,2, \ldots) .
$$

Letting $j$ tend to infinity and observing that $\lim _{n \rightarrow \infty} \xi_{n}=\xi$, this would give us $A(R(\xi))=0$ which contradicts with the fact that $\xi$ is a transcendental formal power series. Therefore the sequence $\left\{A_{n}(y)\right\}_{n=0}^{\infty}$ must have infinitely many different terms. This implies that

$$
\limsup _{n \rightarrow \infty} H\left(A_{n}\right)=\infty .
$$

For otherwise the sequence $\left\{H\left(A_{n}\right)\right\}_{n=0}^{\infty}$ would be bounded above. Since $A_{n}(y)(n=0,1,2, \ldots)$ are polynomials over $K[x]$ with $\operatorname{deg}\left(A_{n}\right)=m$ for sufficiently large $n$ and $K$ is finite, the sequence $\left\{A_{n}(y)\right\}_{n=0}^{\infty}$ would have finitely many different terms, contrary to the fact that $\left\{A_{n}(y)\right\}_{n=0}^{\infty}$ has infinitely many different terms. Thus $\lim \sup H\left(A_{n}\right)=\infty$ must hold. Then the sequence $\left\{H\left(A_{n}\right)\right\}_{n=0}^{\infty}$ has a subsequence $\left\{H\left(A_{n_{i}}\right)\right\}_{i=0}^{\infty} \begin{gathered}n \rightarrow \infty \\ \text { such that }\end{gathered}$

$$
1<H\left(A_{n_{1}}\right)<H\left(A_{n_{2}}\right)<H\left(A_{n_{3}}\right)<\cdots, \quad \lim _{i \rightarrow \infty} H\left(A_{n_{i}}\right)=\infty .
$$

Therefore we infer from (3.5) and $H\left(\gamma_{n_{i}}\right)=H\left(A_{n_{i}}\right)(i=0,1,2, \ldots)$ that

$$
0<\left|A_{n_{i}}(\gamma)\right| \leq H\left(A_{n_{i}}\right)^{-s_{n_{i}}}
$$

for sufficiently large $i$. Since $\operatorname{deg}\left(A_{n_{i}}\right)=m$, this implies that $\gamma$ is a $U$-number with

$$
\mu(\gamma) \leq m
$$


Now we shall show that $\mu(\gamma) \geq m$. If $m=1$, then $\mu(\gamma) \geq m$. Let $m>1$ and $B(y)=$ $b_{0}+b_{1} y+\cdots+b_{g} y^{g}$ be any polynomial over $K[x]$ with $\operatorname{deg}(B)=g$ and $1 \leq g \leq m-1$. Then, for any positive rational integer $\nu$,

$$
B(\gamma)=B\left(\gamma_{\nu}+\rho_{\nu} \delta_{\nu}\right)=B\left(\gamma_{\nu}\right)+\rho_{\nu} \theta_{\nu}
$$

where

$$
\theta_{\nu}=b_{1} \delta_{\nu}+\cdots+b_{g}\left(\left(\begin{array}{l}
g \\
1
\end{array}\right) \gamma_{\nu}^{g-1} \delta_{\nu}+\left(\begin{array}{l}
g \\
2
\end{array}\right) \gamma_{\nu}^{g-2} \rho_{\nu} \delta_{\nu}^{2}+\cdots+\rho_{\nu}^{g-1} \delta_{\nu}^{g}\right) .
$$

Similarly as in the previous paragraph, we obtain for sufficiently large $\nu$

$$
\left|\theta_{\nu}\right| \leq c_{1}^{u_{\nu}} \quad \text { and } \quad\left|\rho_{\nu}\right|\left|\theta_{\nu}\right| \leq c_{1}^{-u_{\nu} s_{\nu}} .
$$

There exists a real constant $c_{2}$ with $0<c_{2}<1$ such that

$$
s_{\nu} \geq c_{2} \frac{u_{\nu+1}}{u_{\nu}}
$$

and hence

$$
\left|\rho_{\nu}\right|\left|\theta_{\nu}\right| \leq c_{1}^{-c_{2} u_{\nu+1}}
$$

hold for sufficiently large $\nu$. Since $\operatorname{deg}\left(\gamma_{\nu}\right)=m$, it follows that $B\left(\gamma_{\nu}\right) \neq 0$. Thus, by Lemma 2.4 and (3.4), we have for sufficiently large $\nu$

$$
\left|B\left(\gamma_{\nu}\right)\right| \geq H(B)^{-(m-1)} H\left(\gamma_{\nu}\right)^{-(m-1)} \geq H(B)^{-(m-1)} c_{1}^{-u_{\nu}(m-1)} .
$$

We choose two real numbers $\lambda$ and $\eta$ such that

$$
\lambda>\frac{2(m-1)}{c_{2}}
$$

and

$$
\eta>\frac{(m-1)(\lambda+1)}{c_{2}}
$$

The inequality

$$
\eta<\frac{u_{\nu+1}}{u_{\nu}}
$$

is verified for sufficiently large $\nu$.

Let $B(y)$ be a polynomial over $K[x]$ with $1 \leq \operatorname{deg}(B) \leq m-1$ and with sufficiently large height $H(B)$. Let $n$ be the unique positive rational integer satisfying

$$
c_{1}^{u_{n}} \leq H(B)<c_{1}^{u_{n+1}} .
$$

If $c_{1}^{u_{n}} \leq H(B)<c_{1}^{u_{n+1} / \lambda}$ holds, then it follows from 3.7, 3.8 and 3.9 for $\nu=n$ that

$$
\begin{aligned}
B(\gamma) & =B\left(\gamma_{n}\right)+\rho_{n} \theta_{n}, \\
\left|B\left(\gamma_{n}\right)\right| & \geq H(B)^{-2(m-1)}, \\
\left|\rho_{n}\right|\left|\theta_{n}\right| & <H(B)^{-\lambda c_{2}} .
\end{aligned}
$$

Then, by $(3.10)$, we obtain $\left|\rho_{n}\right|\left|\theta_{n}\right|<\left|B\left(\gamma_{n}\right)\right|$. So, using the property of non-Archimedean absolute value, that is, $|\alpha+\beta|=\max \{|\alpha|,|\beta|\}$ is verified for $\alpha, \beta \in \mathbb{K}$ when $|\alpha| \neq|\beta|$, we get

$$
|B(\gamma)|=\left|B\left(\gamma_{n}\right)\right| \geq H(B)^{-2(m-1)} .
$$


If $c_{1}^{u_{n+1} / \lambda} \leq H(B)<c_{1}^{u_{n+1}}$ holds, then it follows from 3.7, 3.8, 3.9 and 3.12 for $\nu=n+1$ that

$$
\begin{aligned}
B(\gamma) & =B\left(\gamma_{n+1}\right)+\rho_{n+1} \theta_{n+1} \\
\left|B\left(\gamma_{n+1}\right)\right| & \geq H(B)^{-(m-1)(\lambda+1)} \\
\left|\rho_{n+1}\right|\left|\theta_{n+1}\right| & \leq c_{1}^{-c_{2} u_{n+2}}<c_{1}^{-c_{2} \eta u_{n+1}}<H(B)^{-\eta c_{2}} .
\end{aligned}
$$

Then, by (3.11), we obtain $\left|\rho_{n+1}\right|\left|\theta_{n+1}\right|<\left|B\left(\gamma_{n+1}\right)\right|$. Hence,

$$
|B(\gamma)|=\left|B\left(\gamma_{n+1}\right)\right| \geq H(B)^{-(m-1)(\lambda+1)} .
$$

We infer from 3.13 and 3.14 that

$$
|B(\gamma)| \geq H(B)^{-(m-1)(\lambda+1)}
$$

for all polynomials $B(y)$ over $K[x]$ with $1 \leq \operatorname{deg}(B) \leq m-1$ and with sufficiently large height $H(B)$. This implies that

$$
\mu(\gamma) \geq m
$$

By (3.6) and 3.15, we conclude that $\mu(\gamma)=m$. Then $\gamma=\alpha_{0}+\alpha_{1} \xi+\cdots+\alpha_{k} \xi^{k}$ is a $U_{m}$-number. This completes the proof of Theorem 1.1 .

Remark 3. Using Lemma 2.4 and $(3.4)$, we can rewrite $(3.9)$ exactly as

$$
\left|B\left(\gamma_{\nu}\right)\right| \geq H(B)^{-(m-1)} H\left(\gamma_{\nu}\right)^{-\operatorname{deg}(B)} \geq H(B)^{-(m-1)} c_{1}^{-u_{\nu} \operatorname{deg}(B)} .
$$

If we apply (3.16) in place of $(3.9)$ in the proof of Theorem 1.1. then we see that

$$
|B(\gamma)| \geq H(B)^{-(m-1)-\lambda \operatorname{deg}(B)}
$$

holds true for all polynomials $B(y)$ over $K[x]$ with $1 \leq \operatorname{deg}(B) \leq m-1$ and with sufficiently large height $H(B)$. This yields that

$$
w_{t}(\gamma) \leq m-1+\lambda \cdot t \quad \text { for } \quad t=1, \ldots, m-1
$$

when $m \geq 2$.

Remark 4. Let us denote by $\overline{\mathbb{K}}$ the completion of the algebraic closure of $\mathbb{K}$ with respect to $|\cdot|$. We regard the field $\overline{\mathbb{K}}$ as the analogue of the field $\mathbb{C}$ of complex numbers. The height, the degree and the conjugates of an element $\alpha$ in $\mathbb{K}$, which is algebraic over $K(x)$ are defined exactly as in Subsection 1.1. Due to Bundschuh 3, Mahler's classification is carried from $\mathbb{K}$ to $\overline{\mathbb{K}}$. $A$-, $S$-, $T$-, $U$ - and $U_{m}$-numbers in $\overline{\mathbb{K}}$ are defined as in Subsection 1.2 (We just begin with $\xi \in \overline{\mathbb{K}}$ in place of $\xi \in \mathbb{K}$.) Moreover, we observe that Lemma 2.1. Lemma 2.2. Lemma 2.3 and Lemma 2.4 hold true not only for algebraic formal power series in $\mathbb{K}$ but also for elements in $\mathbb{K}$, which are algebraic over $K(x)$. Then we see that Theorem 1.1 is also valid for the elements $\alpha_{0}, \ldots, \alpha_{k}(k \geq 1)$ in $\overline{K(x)}$ with $\alpha_{k} \neq 0$. (Note that an element of $\overline{K(x)}$ need not be in $\mathbb{K}$, but it is certainly in $\overline{\mathbb{K}}$.) In this case, the occurring $U_{m}$-number is in $\overline{\mathbb{K}}$.

Remark 4 enables us to give the following example to illustrate Theorem 1.1 .

Example 1. Let $m$ be any positive rational integer. In Theorem 1.1, let us take $\alpha_{0}=\cdots=$ $\alpha_{k}=\sqrt[m]{x}$, where $\sqrt[m]{x}$ is defined as a root of the polynomial $y^{m}-x$, and $\xi=\sum_{n=0}^{\infty} x^{-(n+1) !}$. Then $\alpha_{0}+\alpha_{1} \xi+\cdots+\alpha_{k} \xi^{k}$ is a $U_{m}$-number.

Acknowledgement. The author would like to thank the referees for their helpful suggestions. 


\section{ON MAHLER'S CLASSIFICATION OF FORMAL POWER SERIES OVER A FINITE FIELD}

\section{REFERENCES}

[1] ALNIAÇIK, K.: On the subclasses $U_{m}$ in Mahler's classification of the transcendental numbers, İstanbul Üniv. Fen Fak. Mecm. Ser. A 44 (1979), 39-82.

[2] BUGEAUD, Y.: Approximation by Algebraic Numbers, Cambridge Tracts in Math. 160, Cambridge University Press, Cambridge, 2004.

[3] BUNDSCHUH, P.: Transzendenzmasse in Körpern formaler Laurentreihen, J. Reine Angew. Math. 299/300 (1978), 411-432.

[4] CHAVES, A. P.-MARQUES, D.: An explicit family of $U_{m}$-numbers, Elem. Math. 69 (2014), 18-22.

[5] CHAVES, A. P.-MARQUES, D.-TROJOVSKÝ, P.: On the arithmetic behavior of Liouville numbers under rational maps, Bull. Braz. Math. Soc. New Series 52 (2021), 803-813.

[6] KEKEÇ, G.: U-numbers in fields of formal power series over finite fields, Bull. Aust. Math. Soc. 101 (2020), $218-225$.

[7] KEKEÇ, G.: On transcendental formal power series over finite fields, Bull. Math. Soc. Sci. Math. Roumanie 63(111) (2020), 349-357.

[8] LEVEQUE, W. J.: On Mahler's U-numbers, J. London Math. Soc. 28 (1953), 220-229.

[9] MAHLER, K.: Zur Approximation der Exponentialfunktion und des Logarithmus I, II, J. Reine Angew. Math. 166 (1932), 118-150.

[10] MAHLER, K.: Über eine Klasseneinteilung der p-adischen Zahlen, Mathematica (Leiden) 3 (1935), 177-185.

[11] OOTO, T.: The existence of T-numbers in positive characteristic, Acta Arith. 189 (2019), 179-189.

[12] ORYAN, M. H.: Über die Unterklassen $U_{m}$ der Mahlerschen Klasseneinteilung der transzendenten formalen Laurentreihen, İstanbul Üniv. Fen Fak. Mecm. Ser. A 45 (1980), 43-63.

[13] PERRON, O.: Irrationalzahlen, Walter de Gruyter \& Co., Berlin, 1960.

[14] SCHLICKEWEI, H. P.: p-adic T-numbers do exist, Acta Arith. 39 (1981), 181-191.

[15] SCHMIDT, W. M.: T-numbers do exist. In: Symposia Mathematica, Vol. IV (INDAM, Rome, 1968/1969), Academic Press, London, 1970, pp. 3-26.

[16] SPRINDŽUK, V. G.: Mahler's Problem in Metric Number Theory. Transl. Math. Monogr. 25, Amer. Math. Soc., Providence, R.I., 1969.

[17] WALDSCHMIDT, M.: Diophantine Approximation on Linear Algebraic Groups. Grundlehren Math. Wiss. 326, Springer, Berlin-Heidelberg-New York, 2000.

Received 27. 9. 2020

Accepted 31. 3. 2021
Department of Mathematics

Faculty of Science

Istanbul University

34134 Vezneciler, Fatih, Istanbul TURKEY

E-mail: gulkekec@istanbul.edu.tr 